\title{
A solar cycle of spacecraft anomalies due to internal charging
}

\author{
G. L. Wrenn, D. J. Rodgers, and K. A. Ryden \\ QinetiQ, A8, Cody Technology Park, Farnborough, Hampshire, GU14 OLX, UK
}

Received: 22 October 2001 - Revised: 27 February 2002 - Accepted: 25 March 2002

\begin{abstract}
It is important to appreciate how the morphology of internal charging of spacecraft systems, due to penetrating electrons, differs from that of the more common surface charging, due to electrons with lower energy. A specific and recurrent anomaly on a geostationary communication satellite has been tracked for ten years so that solar cycle and seasonal dependencies can be clearly established. Concurrent measurements of sunspot number, solar wind speed and 2-day $>2 \mathrm{MeV}$ electron fluence are presented to highlight pertinent space weather relationships, and the importance of understanding the complex particle interaction processes involved.
\end{abstract}

Key words. Magnetospheric physics (energetic particles; trapped; solar wind - magnetosphere interactions) - space plasma physics (spacecraft sheaths, wakes, charging)

It is not uncommon for satellites in orbit to exhibit some irregularity in performance, and operators apply rigorous procedures for logging and examining these "anomalies". The concept of space weather has recently gained common acceptance with the realisation that the performance of many technological systems on Earth and in space are strongly influenced by the dynamic nature of the space environment. Disruption of spacecraft operations is one of the most significant effects. Electrostatic charging of materials has long been recognised as a serious space hazard, due to the damaging electrostatic discharge (ESD) that can be triggered. It is always important to minimise surface charging caused by the build-up of $<100 \mathrm{keV}$ electrons that impact but do not penetrate the spacecraft surface. However, for many orbits (e.g. GEO synchronous missions) the significance of highly energetic electrons must also be addressed; these can penetrate the surface and accumulate within internal dielectrics, such

Correspondence to: G. L. Wrenn

(gordonwrenn@tiscali.co.uk) as printed circuit boards or cable insulation. When internal dielectrics become charged, any subsequent breakdown is likely to have more serious consequences. Numerous spacecraft systems have proved to be susceptible to this process, to a larger or smaller extent, but some of the latter have fortuitously provided ideal data for developing an understanding of the charge-discharge process that is often a consequence of what might be termed "inclement" weather.

Here we report on a specific anomaly that has occurred over two hundred times during a ten-year period, effectively covering a whole solar cycle, a dominant driver for many space weather processes. This repeated mode of switching on a GEO communications satellite has been reported (Wrenn, 1995), together with evidence for establishing internal charging as the cause. The ESD sensitivity of the affected circuitry was obviously not a design feature. It appears to have originated during the "unique and truly amazing" event (Blake et al., 1992) in March 1991, but it has yielded a rare demonstration of environmental cause and engineering effect.

The maximum of the smoothed sunspot number for cycle 22 was in July 1989; the minimum in May 1996, then heralded as the start of cycle 23, which peaked in April 2000. Each day of the years 1991 through 2000 is displayed in Fig. 1 as a traffic light presentation based on the 2-day fluences of $>2 \mathrm{MeV}$ electrons measured at geostationary GOES satellites. The days are ordered by 27.4-day Carrington solar rotations, starting with 1837 and ending with 1971; the righthand panel plots the smoothed sunspot number on a scale from 0 to 180 . Black spots mark those days on which the mode switching anomalies occurred. The long-term repetition of the phantom command is surprising, because electrostatic discharge is basically a chaotic process but more importantly, the operational impacts have always been minor and far from the catastrophic failures that have degraded and terminated some other missions. The SEM (Space Environment Monitor) instrument on GOES-7 operated for 9 years and the measurements are coded as follows: 


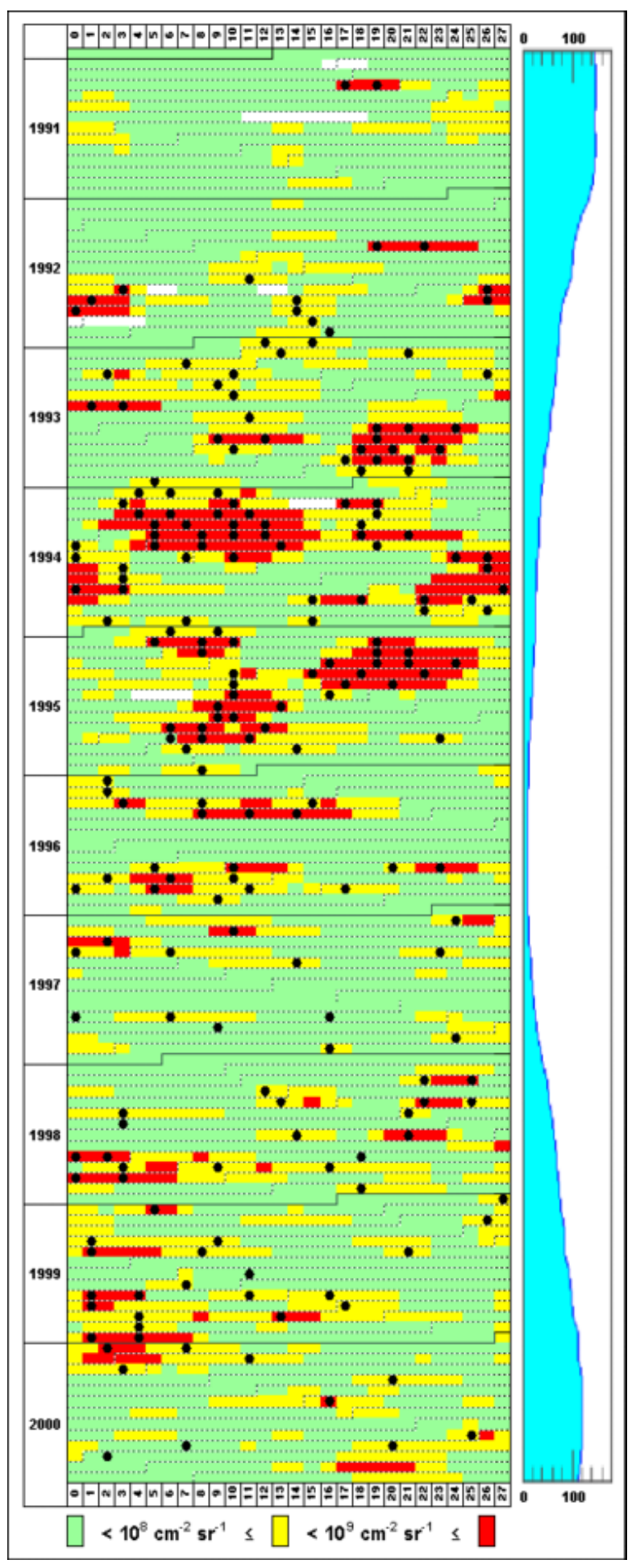

Fig. 1. (a) 2-day fluence of $>2 \mathrm{MeV}$ electrons at GEO showing 214 correlated phantom commands. (b) smoothed sunspot number from January 1991 to December 2000.

- Red: 2-day fluence $\geq 10^{9} \mathrm{~cm}^{-2} \mathrm{sr}^{-1}$,

- Amber: $10^{9}>2$-day fluence $\geq 10^{8}$,

- Green: 2-day fluence $<10^{8} \mathrm{~cm}^{-2} \mathrm{sr}^{-1}$,

- White: no GOES data.
Table 1. Days with data and switches within the cycle [* GOES-7 equivalent $\left.\left(\mathrm{cm}^{-2} \mathrm{sr}^{-1}\right)\right]$

\begin{tabular}{ccccc}
\hline & $\begin{array}{c}\text { 2-day } \\
\text { fluence* }\end{array}$ & $\begin{array}{c}\text { Data } \\
\text { days }\end{array}$ & $\begin{array}{c}\text { ESD } \\
\text { switches }\end{array}$ & $\begin{array}{c}\text { Switch } \\
\text { days }\end{array}$ \\
\hline Green & $<10^{8}$ & 2291 & 7 & $0.3 \%$ \\
Amber & $10^{8}-10^{9}$ & 952 & 102 & $10.7 \%$ \\
Red & $\geq 10^{9}$ & 332 & 105 & $31.6 \%$ \\
& Total & 3575 & 214 & $6.0 \%$ \\
\hline
\end{tabular}

In order to extend the analysis beyond March 1996, it has been necessary to utilise measurements from a similar detector on GOES-8; this has involved the introduction of a normalisation factor, to take into account sensitivity and longitude differences (Wrenn, 1995).

The outer belt electron enhancements (OBEEs) tend to last for several days but often exhibit a 27-day recurrence that reflects the persistence of coronal holes on the Sun. Their occurrence peaks not at solar maximum, but during the declining phase when high-speed streams of solar wind are more stable and long-lived. This is highlighted in Fig. 2 which shows plots of the 27-day maxima for both the $>2 \mathrm{MeV}$ electron fluence and solar wind speed, through the cycle from July 1989 to April 2000, together with the smoothed sunspot number. Although there is no direct correlation, the long-lived high-speed streams do occur during 1994 and 1995, approaching solar minimum, but not near solar maximum. A few bursts and associated OBEEs are obviously non-recurrent and appear to be associated with solar proton events, or perhaps coronal mass ejections. This solar cycle pattern fits well with earlier measurements made during cycle 21 (Baker et al., 1993).

The amber level was selected as the apparent threshold for the anomaly (Wrenn and Smith, 1996) and it succeeds in highlighting the clear correlation between the observed switchings and the periods of high $>2 \mathrm{MeV}$ fluence (since September 1997 a few exceptions have been logged). Table 1 details this by giving a breakdown of the 214 "switch-days" within the ten-year ( $3653 \mathrm{~d}$ ) period within the three fluence bands. Given that there is a large displacement in local time between a GOES and the affected spacecraft, the correlation is quite remarkable. The strong link to penetrating electrons shows conclusively that these dayside anomalies are due to internal charging. While surface charging can produce similar effects, these are mainly confined to the nightside because the morphology of $\mathrm{keV}$ electrons is very different with many intensifications occurring around solar maximum, when geomagnetic storms and substorms are most frequent; Wrenn and Smith (1996) utilised Marecs-A anomaly data to illustrate this contrast.

Two-day fluence is used because even with the highest flux, a charging time of more than $30 \mathrm{~h}$ has been required to trigger the breakdown analysed here (Wrenn and Sims, 1996); accordingly, the percentage of red days exhibiting 


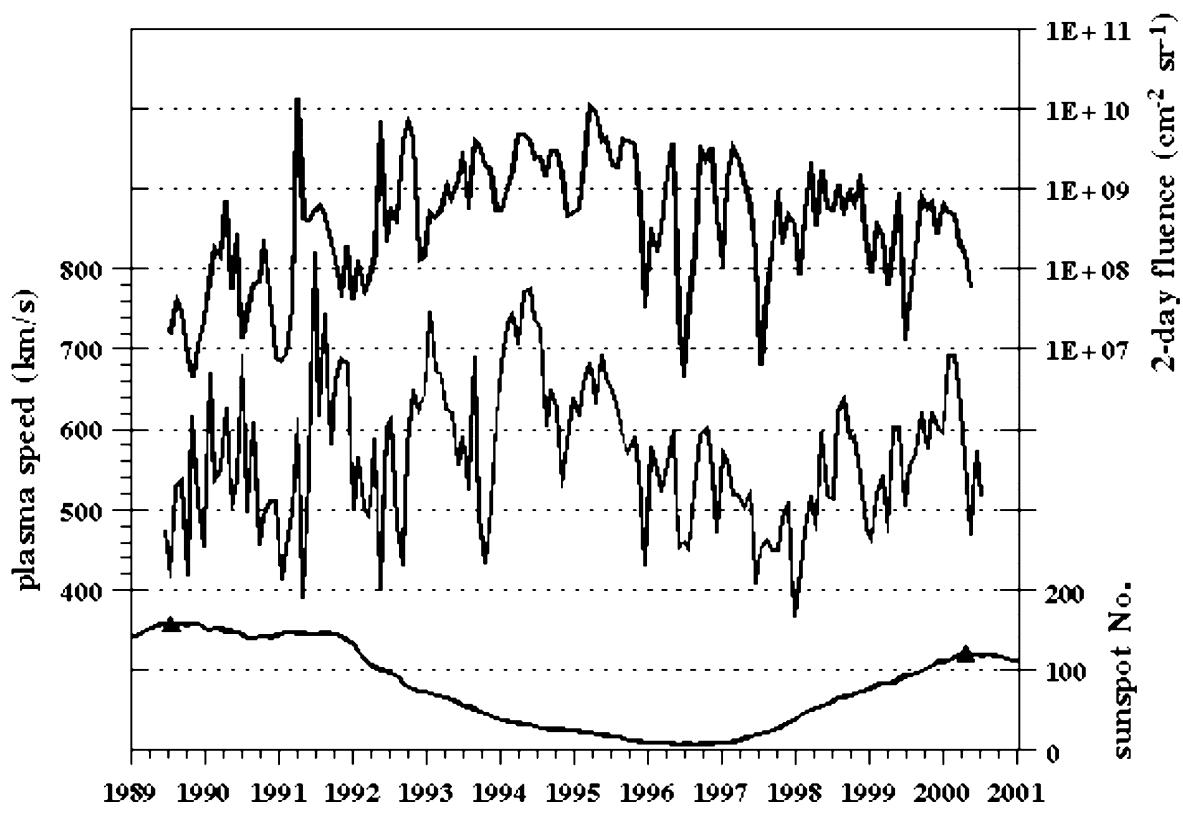

Fig. 2. Profile of peak 2-day $>2 \mathrm{MeV}$ electron fluence compared to smoothed sunspot number and solar wind speed through the complete solar cycle, July 1989 to April 2000.

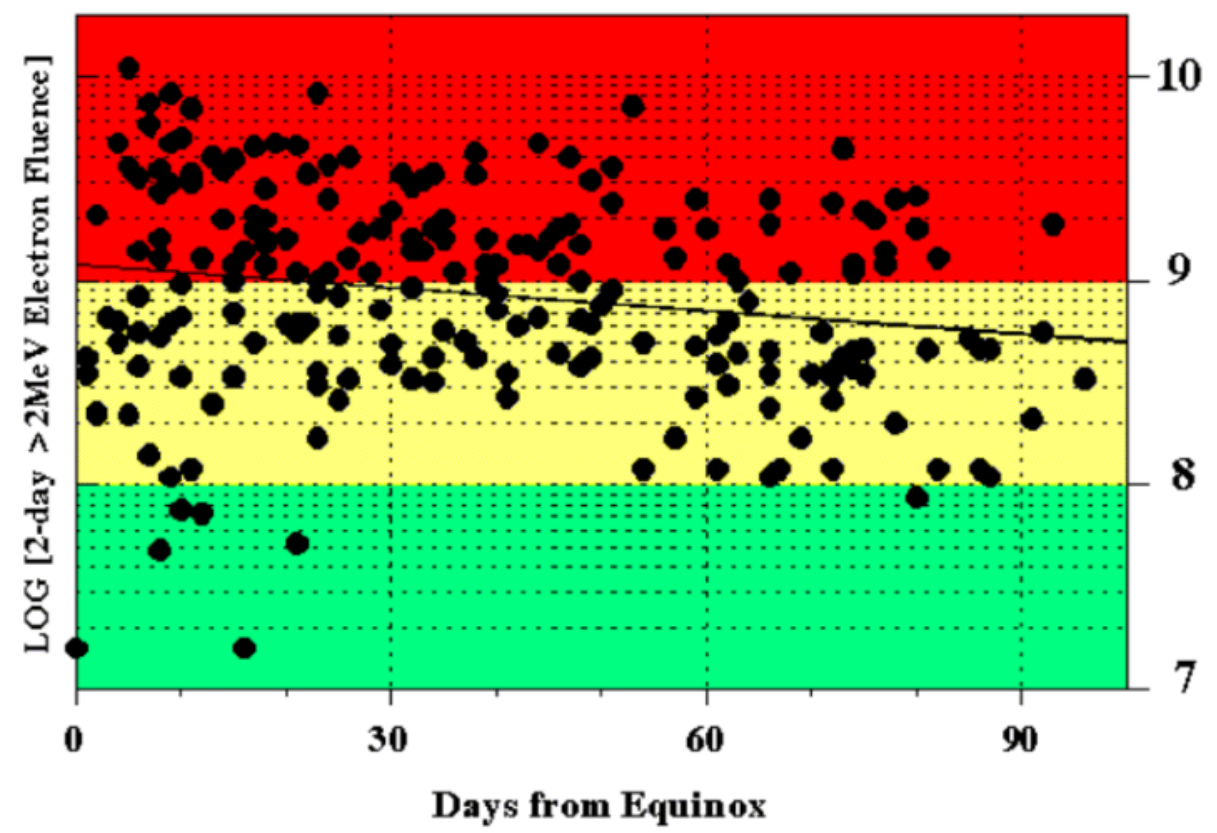

Fig. 3. Distribution of switches with respect to 2-day $>2 \mathrm{MeV}$ electron fluence and to number of days from equinox.

these switches could not be more than $50 \%$. In general, ESD frequency will be a function of the shielding configuration of the charge site and its dielectric properties. Figure 3 reinforces the main message by showing the distribution of anomalies with respect to fluence, but it also explores the significance of season by plotting the switches against displacement from equinox (the line is a simple linear fit). Since coupling between the solar wind and the magnetosphere is easier near equinox, the electron fluences are gen- erally higher and ESD occurrence frequency can be expected to increase. An important implication is that the monitoring of daily $>2 \mathrm{MeV}$ electron fluence at a single location can provide a reliable barometer of the internal charging hazard in GEO. That the recent cluster of switches at low fluence is near equinox might indicate a different trigger, perhaps associated with eclipse effects, due to some long-term material degradation.

A combination of circuit modification and adjustment of 
the shielding arrangements near the suspected charge site achieved mitigation of the engineering problem. Consequently, follow-on missions have been less interesting from a space weather perspective. Terrestrial weather can be generally analysed in terms of "climate" with the superposition of "storm" perturbations. Solar cycle and season, combined with geomagnetic location ( $L$-value), supply here an equivalent and appropriate framework. The population of penetrating electrons in OBEEs is characterised by spatial distribution, flux intensity and spectral "hardness". The broad features have now been modelled (Wrenn et al., 2000) as part of the DICTAT engineering analysis tool that is available within the space environment information system (SPENVIS) on the web at http://www.spenvis.oma.be. For satellite designers, Fig. 1 is simply a reminder to focus on countering the internal charging hazard as the next solar minimum approaches but, for magnetospheric physicists, it should be a spur to gain a much needed understanding of the electron acceleration and trapping processes involved.

Acknowledgements. The GOES data are supplied by the NOAA Space Environment Center, Boulder, USA; T. Onsager there provided important guidance on interpretation. The NSSDC OMNIWeb database was accessed to download the solar wind speed data.
The Editor in chief thanks a referee for his help in evaluating this paper.

\section{References}

Baker, D. N., Goldberg, R. A., Herraro, F. A., Blake, J. B., and Callis, L. B.: Satellite and rocket studies of relativistic electrons and their influence on the middle atmosphere, J. Atmosph. and Terr. Phys., 55, 1619-1628, 1993.

Blake, J. B., Gussenhoven, M. S., Mullen, E. G., and Fillius, R. W.: Identification of an unexpected space radiation hazard, IEEE Trans. Nucl. Sci., 39, 1761, 1992.

Wrenn, G. L.: Conclusive evidence for internal dielectric charging anomalies on geosynchronous communications spacecraft, J. Spacecraft and Rockets, 32, 514-520, 1995.

Wrenn, G. L. and Sims, A. J.: Internal charging in the outer zone and operational anomalies, Radiation Belts: Models and Standards, AGU Geophysical Monograph 97, 275-278, 1996.

Wrenn, G. L. and Smith, R. J. K.: Probability factors governing ESD effects in geosynchronous orbit, IEEE TNS, 43, 27832789, 1996.

Wrenn, G. L., Rodgers, D. J., and Buehler, P.: Modeling the outer belt enhancements of penetrating electrons, J. Spacecraft and Rockets, 37, 408-415, 2000. 